\title{
Expected-Reliability Analysis for Wireless CORBA with Imperfect Components
}

\author{
Xinyu Chen and Michael R. Lyu \\ Department of Computer Science and Engineering \\ The Chinese University of Hong Kong, Shatin, N.T., Hong Kong \\ \{xychen,lyu\}@cse.cuhk.edu.hk
}

\begin{abstract}
Reliability analysis has long been an important area of research for wired networks. However, little reliability analysis has been conducted on wireless networks. Wireless networks, such as wireless CORBA, inherit the unique handoff characteristic which leads to different communication structures with various types and numbers of components and links. Therefore, the traditional definition of two-terminal reliability is not applicable anymore. We propose a new term, two-terminal expected-reliability, to integrate those different communication structures into one metric, which includes not only the failure parameters but also the service parameters. Nevertheless, the two-terminal expected-reliability is still a monotonically decreasing function of time $t$. The expected-reliability and the corresponding MTTF are evaluated quantitatively in different communication schemes. To observe the gains in reliability improvement, the reliability importances of imperfect components are also evaluated. The results show that the failure parameters of different components take different effects on the MTTF and on the reliability importance. With different expected working times of a system, the focus of reliability improvement should be transferred to different components. Although our analysis is conducted on wireless CORBA platforms, it is extensible to generic wireless network systems.

Keywords: Two-terminal expected-reliability, Wireless CORBA, Imperfect components, Reliability importance, Handoff
\end{abstract}

\section{Introduction}

Reliability analysis has long been an important area of research for wired networks $[1,7,8,17,18]$ but not for wireless networks. As the mobile technology matures, however, wireless networks $[6,10]$ are being used in more applications in providing significant benefits to mobile users. Wireless networks are more prone to failures and loss of ac- cess; therefore, the reliability requirements of wireless networks should be rigorously assessed. The reliability issue for wireless networks is quite different from that for wired networks as the terminal mobility feature is unique in wireless networks.

Object Management Group (OMG) has published a wireless CORBA (Common Object Request Broker Architecture) specification to provide wireless access and terminal mobility in CORBA [13]. As Figure 1 shows, a wireless

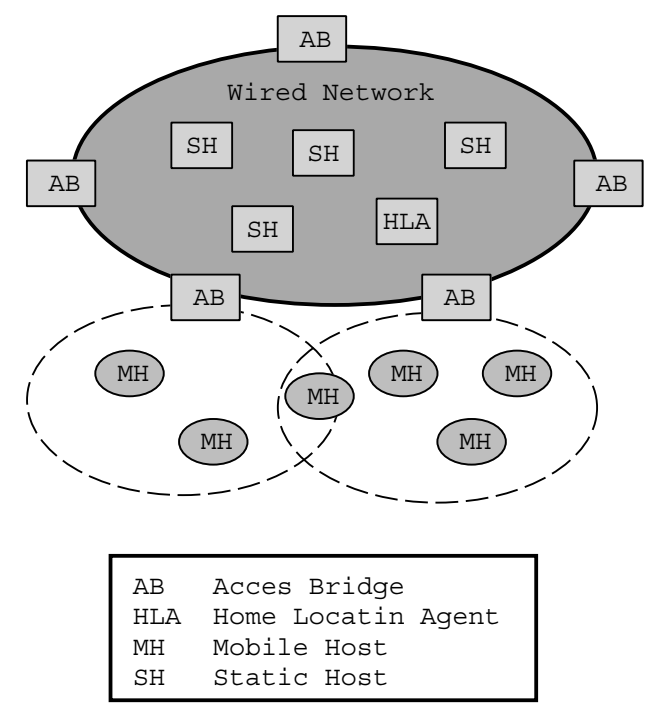

Figure 1. Wireless CORBA environments and components

CORBA environment consists of four main components excluding links:

- A Mobile Host (MH) is a terminal which is equipped with a wireless interface and keeps network connections while roaming in wireless networks;

- A Static Host (SH) is a normal and fixed node in wired networks; 
- An Access Bridge (AB) sits between MHs and SHs to relay messages for its associated $\mathrm{MHs}$, which is deployed in wired networks but contains a wireless interface;

- A Home Location Agent (HLA) keeps track of the current location of its registered MHs and provides operations to query an MH's location.

In wireless $\mathrm{CORBA}$, an $\mathrm{AB}$ connects to the wired network from a fixed location using standard cabling. It receives, buffers and transmits messages between the wireless network and the wired network infrastructure. A single $A B$ supports a group of MHs and functions within a range, providing a single cell of wireless coverage. Multiple ABs provide multiple cells, allowing MHs to roam from one cell to another while maintaining connections to the network. This process is called handoff. MHs communicate with each other only via ABs. No messages can be exchanged directly between MHs, even if they stay in the same cell of an AB.

For the wireless CORBA to be functional, its components must be fit for service. Unfortunately, this is not always the case, because these components may not be reliable [7]. We need a mechanism to assess the reliability of wireless networks. However, as the wireless CORBA provides the handoff operation which is a new feature, the traditional two-terminal reliability [19] is not suitable anymore. The handoff operation causes the existing communication structure to change with the MH's movement. At different time periods, different components are employed. This paper seeks a new approach to define the reliability metric in wireless networks, which not only keeps the monotonically decreasing characteristic of reliability but includes the mobility nature in the system. Different effects imposed by component failure parameters and mobile service parameters will be given through numerical examples. To observe the gains in reliability improvement, the reliability importances of imperfect components are also evaluated.

\section{Related Work}

Much work has been done in studying the reliability of wired networks $[8,16,17,18]$. Aggarwal et al. [1] extended the reliability evaluation of communication systems with imperfect nodes by utilizing a concept that the failure of a node implies the failure of links connecting it. Torrieri [21] proposed another method that compensates for unreliable nodes in network reliability computation, the cost of which increases linearly with the number of links. Netes and Filin [11] added the imperfect nodes into paths for decomposing the network directly into an event-tree. Ke and Wang [7] also exploited some partition techniques to directly compute the network reliability expression instead of using any compensating methods.
Most recently, some work has been conducted in providing fault tolerance in wireless environments for reliability engineering. Neves and Fuchs [12] proposed a coordinated checkpointing protocol for wireless distributed systems. Park and Yeom developed an asynchronous recovery scheme based on optimistic message logging [14]. Chen and Lyu [4] presented a message logging and recovery strategy in wireless CORBA. Alagra et al. [2] utilized normal replication strategies to tolerate mobile support station failures. Biaz and Vaidya [3] discussed to tolerate location register failures by the fault-tolerant forwarding strategy and the two-path forwarding strategy.

The analysis of performance and reliability issues in wireless networks has been addressed only by a handful of researchers. Pradhan et al. [15] discussed the design and trade-off in recoverable mobile environments. Performance and effectiveness analysis of checkpointing was conducted in [5]. Reliability and survivability issues of wireless networks were discussed in [20], which concluded that each component engaged in the end-to-end connection is a potential point of failure. However, it did not explicitly state how the user mobility, which is unique in wireless networks, affects the end-to-end reliability. Varshney et al. [22] proposed a scalable approach utilizing Wireless Infrastructure Building-block (WIB) to model and simulate the reliability and the survivability of infrastructure-oriented wireless networks, in which constant component hazard rates were adopted. It is apparent that more research activities in investigating fault tolerance and reliability engineering techniques for wireless networks should be conducted.

\section{Definitions and Assumptions}

In general, reliability is defined as the probability that a system performs its intended functions successfully for a given period of time under specified environmental conditions, and we call the probability of successful communication between the source node and the target node as twoterminal reliability [19]. For two nodes to communicate with each other, there should be at least one operating path between them. An operating path indicates that all the intermediate nodes and links of the path should be in operation states. A node is operational if and only if it functions as intended, and a link is operational if and only if it allows communication from its source node to its terminal node [21]. Because the two-terminal reliability problem in wired networks has been studied thoroughly in the literature, we assume that the intermediate nodes and wired links are always reliable, i.e., there will always be a reliable path between an $\mathrm{AB}$ and an $\mathrm{SH}$ or between an $\mathrm{AB}$ and another $\mathrm{AB}$. For the wireless part, an $\mathrm{MH}$ has only one wireless link with one $\mathrm{AB}$ and it associates with only one $\mathrm{AB}$ at a time except during handoff. Therefore, the communication path built on 
the top of wireless links is simple, and we assume that the wireless link failures are negligible. However, all the four components of wireless CORBA are failure-prone and they can fail independently. Based on the assumptions made before, a successful communication between two nodes is defined as all the engaged nodes, including the source node and the target node, are in the operation states. As a result, the two-SH reliability is the multiplication between the two individual SHs' reliabilities. If one or both of the two terminals are MHs, the traditional two-terminal reliability metric cannot correctly describe the characteristic introduced by the handoff. As MHs move and perform handoff operations, the communication structures will be different. Each communication structure can be regarded as a serial system composed of different types and numbers of engaged components. Additionally, the handoff operation induces that the MH's published address will be outdated and a mechanism is needed to resolve the current location of the $\mathrm{MH}$. Therefore we propose a new term, two-terminal expectedreliability, to address these unique cases in wireless environments. We define the system state, $s$, as the communication structure; therefore, $s$ changes with time $t$. Let $Q_{s}(t)$ denote the probability that the system is in state $s$ at time $t$. The two-terminal expected-reliability at time $t, E R(t)$, is given by

$$
E R(t)=\sum_{s} Q_{s}(t) R_{s}(t)
$$

in which $R_{s}(t)$ denotes the reliability of the system in state $s$ at time $t . R_{s}(t)$ can be expressed by

$$
R_{s}(t)=\prod_{i=1}^{n(s)} R_{i}(t)=\prod_{c}\left[R_{c}(t)\right]^{k_{c}(s)},
$$

in which $n(s), n(s)=1,2, \ldots$, is the number of engaged components in system state $s, R_{i}(t)$ is the reliability of the $i^{t h}$ component, $c$ is the type of a component, which may take a value of $m h, a b, s h$, or $h l a$, and $k_{c}(s), k_{c}(s)=$ $0,1,2, \ldots$, is the number of component $c$ employed in state $s$. $E R(t)$ is a function composed not only of failure parameters but also of service parameters introduced by state probability $Q_{s}(t)$. From the above definitions, we note that the two-terminal expected-reliability can be simply extended to include the reliability metrics of wired and wireless links. The two-SH reliability can be treated as a special case in which the system contains only one communication structure, i.e., $Q_{s}(t)=1$, and $E R(t)=R_{s}(t)=\left[R_{s h}(t)\right]^{2}$. Under the adopted assumptions, we can say that the expectedreliability is a generalization of the traditional two-terminal reliability. Accordingly, we define the two-terminal MTTF as

$$
M T T F=\int_{0}^{\infty} E R(t) d t .
$$

There exist four communication schemes if random communications occur between $\mathrm{MHs}$ and SHs, which are
SS scheme, MS scheme, SM scheme, and MM scheme. In these notations the former capital letter denotes the type of the source node and the latter letter denotes the type of the target node, where $M$ stands for $\mathrm{MH}$ and $S$ stands for $\mathrm{SH}$.

During communications, an $\mathrm{MH}$ associates with an $\mathrm{AB}$ and exchanges messages with other nodes. As the $\mathrm{MH}$ moves, it will make handoffs and associate with new ABs. The sojourn time with an $\mathrm{AB}$ and the handoff completion time are assumed as random variables which are exponentially distributed with parameters $\rho$ and $\eta$, respectively. We also assume that the component hazard rates are constant, i.e., we model component failures as homogeneous Poisson processes, resulting in independent and exponential component failure arrival processes [22]. The constant failure parameters for the four components of wireless CORBA, $\mathrm{MH}$, $\mathrm{AB}, \mathrm{SH}$, and HLA, are $\alpha, \beta, \gamma$, and $\delta$, respectively. Utilizing the exponential distribution as the service distribution and the failure distribution is for simplicity; however, what the failure distribution really is should not affect the conclusions we derived.

\section{Two-Terminal Expected-Reliability and MTTF Analysis}

Different communication schemes engage various types and numbers of components which result in different twoterminal expected-reliabilities and MTTFs. The SS scheme is trivial and its expected-reliability has been derived in the last section, $E R_{s s}(t)=\left[R_{s h}(t)\right]^{2}$. Therefore, we will discuss the remaining MS, SM, and MM schemes in the following three subsections separately.

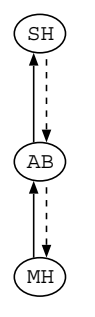

(a)

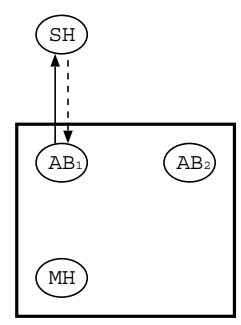

(b)

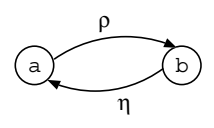

( I )

\section{Figure 2. System states and Markov model in MS scheme}

\subsection{MS Scheme}

The MS scheme is a communication scheme in which an $\mathrm{MH}$ initiates the communication with an $\mathrm{SH}$. Initially, the $\mathrm{MH}$ sends requests over a wireless link, then the associated $\mathrm{AB}$ relays the request messages to the target $\mathrm{SH}$ 
through wired links. After a random sojourn time in the current $\mathrm{AB}$, the $\mathrm{MH}$ may perform a handoff during which the new $\mathrm{AB}$ and the old $\mathrm{AB}$ both should work. The system states are thus shown in Figure 2, in which the solid arrow lines denote the request paths and the dashed arrow lines denote the reply paths. The detailed communication paths among the $\mathrm{MH}, \mathrm{AB}_{1}$, and $\mathrm{AB}_{2}$ in state $b$ are omitted for simplicity, which are different between the network initiated handoff and the terminal initiated handoff [13]; however, the engaged nodes are the same. Furthermore, the detailed communication paths in handoff do not influence the expected-reliability. In addition, the HLA of this MH is also excluded from state $b$. As we know, during the handoff the new $\mathrm{AB}$ sends a location update message to the HLA to inform it that the $\mathrm{MH}$ has changed its associated $\mathrm{AB}$. Normally, the HLA should be in work during a handoff. However, if we employ a simple message retry strategy, the HLA will eventually receive the location update message no matter whether it works or not during the handoff. This is a simple approach to improve the system reliability. After the handoff, the system returns to state $a$ for normal communications. Figure 2(I) shows the Markov model of the system state transition, where $\rho$ is the handoff rate and $\eta$ is the handoff completion rate.

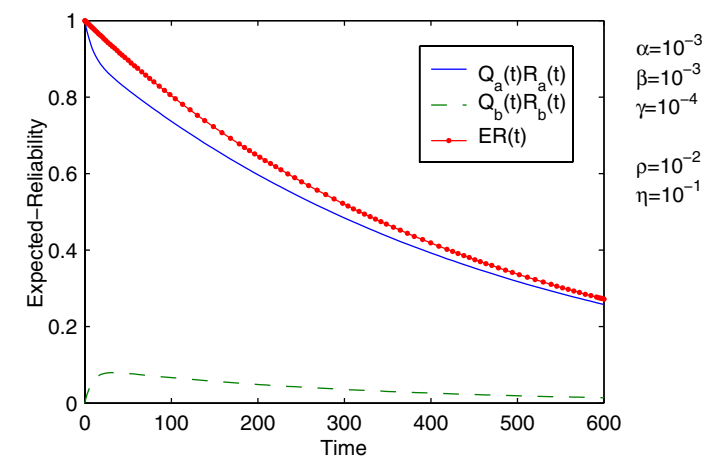

\section{Figure 3. Expected-reliability of MS scheme}

The probabilities of the system in state $a$ and $b$ at time $t$ can be solved analytically, which are given by [9]

$$
Q_{a}(t)=\frac{\eta}{\rho+\eta}+\frac{\rho}{\rho+\eta} \cdot e^{-(\rho+\eta) t}
$$

and

$$
Q_{b}(t)=\frac{\rho}{\rho+\eta}-\frac{\rho}{\rho+\eta} \cdot e^{-(\rho+\eta) t}
$$

One realization of the two-terminal expected-reliability of the MS scheme, $E R_{m s}(t)$, is shown in Figure 3. Different classes of components experience different levels of failures. The SHs are generally more reliable than the MHs and the ABs. Therefore we let $\alpha=\beta=10^{-3}$ and $\gamma=10^{-4}$. Actually, the specific values of the parameters do not change the shapes of the curves. As expected, the probability of the system in state $a$ is much greater than that in state $b$ as the handoff procedure is completed very quickly, resulting in the case that the reliability of state $a$ contributes more to the expected-reliability than that of state $b . R_{b}(t)$ is a monotone decreasing function of time $t$; however, $Q_{b}(t)$ increases first and then approaches an upper limit. All these lead $Q_{b}(t) R_{b}(t)$ to increase first and then decrease. Nevertheless, the expected-reliability $E R(t)$ is still a monotone decreasing function of time $t$. Figure 4 shows the two-
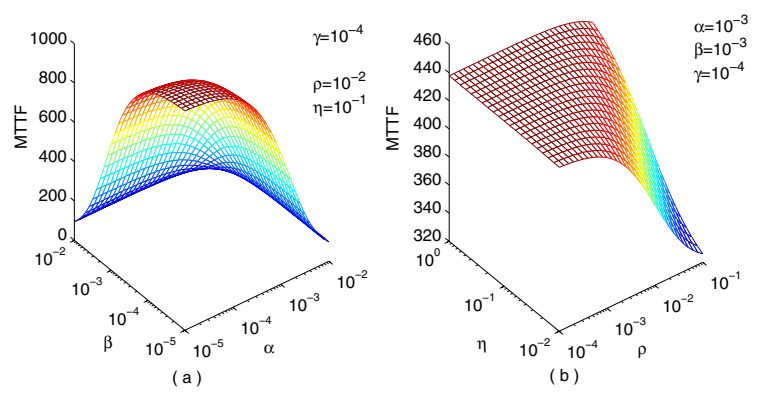

\section{Figure 4. Two-terminal MTTF of MS scheme}

terminal MTTF as a function of failure parameters and state transition parameters. The more reliable the components are, the longer the MTTF is. However, the improvement gains (in terms of the MTTF) reduce with the increase in the failure parameters, $\alpha$ and $\beta$, beyond a certain threshold, which can be observed from Figure 4(a). Such diminishing gains should be carefully considered against the cost of increasing components' reliabilities beyond a limit [22]. This result is also applied to parameter $\gamma$. From the following equation

$$
E R_{m s}(t)=Q_{a}(t) e^{-(\alpha+\beta+\gamma) t}+Q_{b}(t) e^{-(\alpha+2 \beta+\gamma) t},
$$

we see that $\alpha$ and $\gamma$ have the same effects on the $E R_{m s}(t)$ and little difference exists between $\alpha$ and $\beta$ when $Q_{b}(t)$ is much smaller than $Q_{a}(t)$. As the result, Figure 4(a) is almost symmetric. This means that each component is critical to successful system communications. Figure 4(b) shows that when $\rho$ is high, the MTTF increases with $\eta$; however, when $\rho$ is low, the MTTF varies little with $\eta$. This indicates that when the handoff happens frequently, the time spent in the handoff period is very critical to the MTTF, because the reliability is clearly lower in the handoff state $b$ than in the normal state $a$. When $\rho$ is low, however, the contribution of the second term in Equation (6) is small, leading to little change of the MTTF with $\eta$. To achieve a higher expected-reliability, then, MHs experiencing high handoff rates should complete the handoff operation as fast as they can. 


\subsection{SM Scheme}

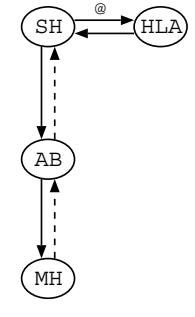

(c)

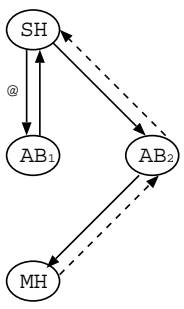

(f)

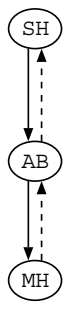

(d)

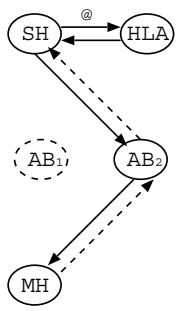

(g)

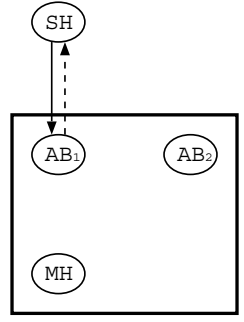

(e)

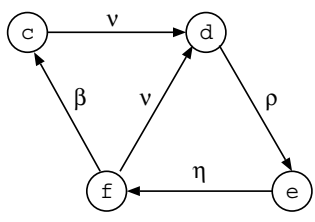

(II)
Figure 5. System states and Markov model in SM scheme

In the SM scheme, an SH initiates communications with an $\mathrm{MH}$. The difference with the MS scheme is that the SM scheme introduces a mechanism to locate the $A B$ with which the MH is currently associated. The location mechanism complicates the system states, as shown in Figure 5. An object on an $\mathrm{MH}$ publishes its Mobile Interoperable Object Reference (MIOR) with the address of the MH's HLA. When an SH first invokes an object on an $\mathrm{MH}$ with a published MIOR, the request message will be sent to the indicated HLA and the HLA will reply with a GIOP (General Inter-ORB Protocol) LOCATION_FORWARD message returning another MIOR indicating the $\mathrm{AB}$ with which the HLA believes the MH is currently associated [13]. This is the system state $c$. The time spent in this state is also assumed as an exponentially distributed random variable with parameter $\nu$. If the $\mathrm{SH}$ receives the LOCATION_FORWARD message from the HLA, the system enters state $d$, a normal communication state. Then, the $\mathrm{MH}$ resends the request to the $\mathrm{AB}$ and the $\mathrm{AB}$ forwards the message to the $\mathrm{MH}$. State $e$ is the handoff state. As the SH does not know whether its target $\mathrm{MH}$ has experienced a handoff or not, it still sends requests to the known $\mathrm{AB}$ as normal despite of the movement of the $\mathrm{MH}$. However, the contacted $\mathrm{AB}$ knows that the $\mathrm{MH}$ has been associated with another $\mathrm{AB}$, and replies to the requests with LOCATION_FORWARD messages returning new MIORs indicating the $\mathrm{AB}$ that the $\mathrm{MH}$ is now associated with, which is state $f$. Because $A B_{1}$ functions as an HLA when the system is in state $f$, the HLA could be treated as a hot standby component to replace $A B_{1}$ when $A B_{1}$ is failed, even though the failure rates may be different between these two components. If $A B_{1}$ fails to reply to a request, the SH may resend the request to the HLA to get the up-to-date MIOR. This is the system state $g$, in which the dashed oval implies that the component is failed. The Markov model of the system state transition is shown in Figure 5(II) in which state $g$ is treated as state $c$ as their communication structures are the same. As state $f$ is also a location-forwarding state, we assume that the time spent in this state follows the same distribution as that in state $c$.

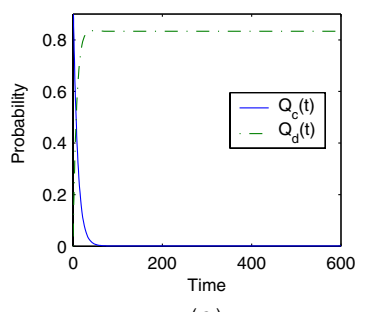

(a)

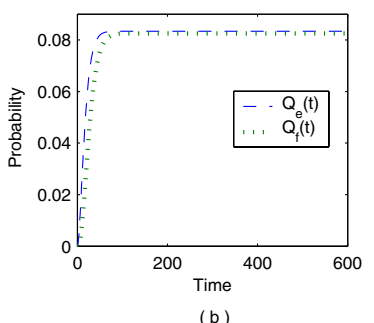

(b)

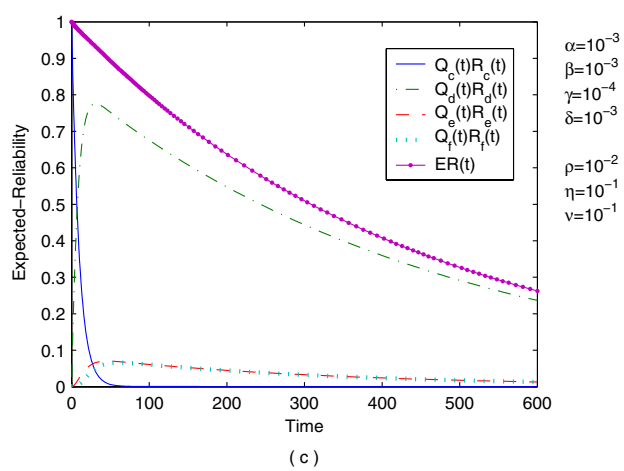

Figure 6. State probability and expectedreliability of SM scheme

The symbolic expression of the probabilities of the system in different states at time $\mathrm{t}$ are difficult to be derived. Therefore we utilize numerical approach here to express their variations with time $t$. Figure 6 shows the result. The corresponding expected-reliability and the MTTF are shown in Figure 6(c) and Figure 7, respectively. In Figure 6 we see that the state $c$ 's contribution to the expectedreliability decreases quickly as time moves on, because the probability of state $c$ diminishes quickly. States $e$ and $f$ assume almost the same probability and consist of the same components; therefore, their contributions to the expectedreliability is almost identical. States $d, e$ and $f$ exhibit similar behaviors in expected-reliability as all these three states show similar curve shapes in state probability. When the decrease of the state reliability cancels out the increase of the state probability, the state's contribution to the expected- 

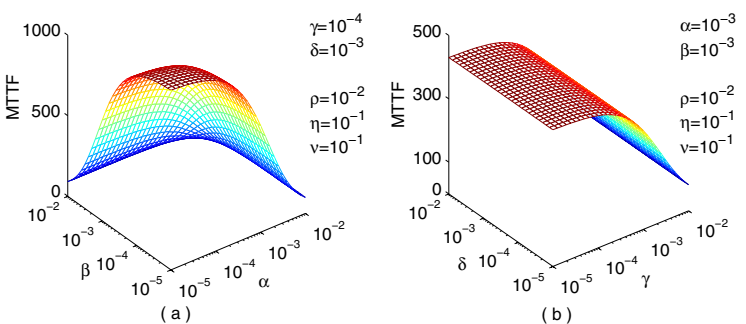

(b)
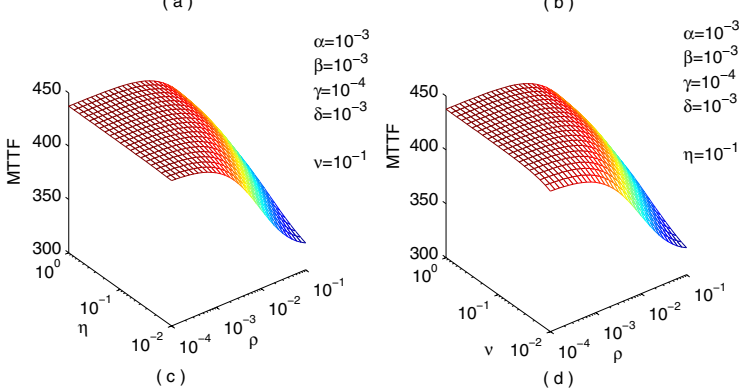

(d)
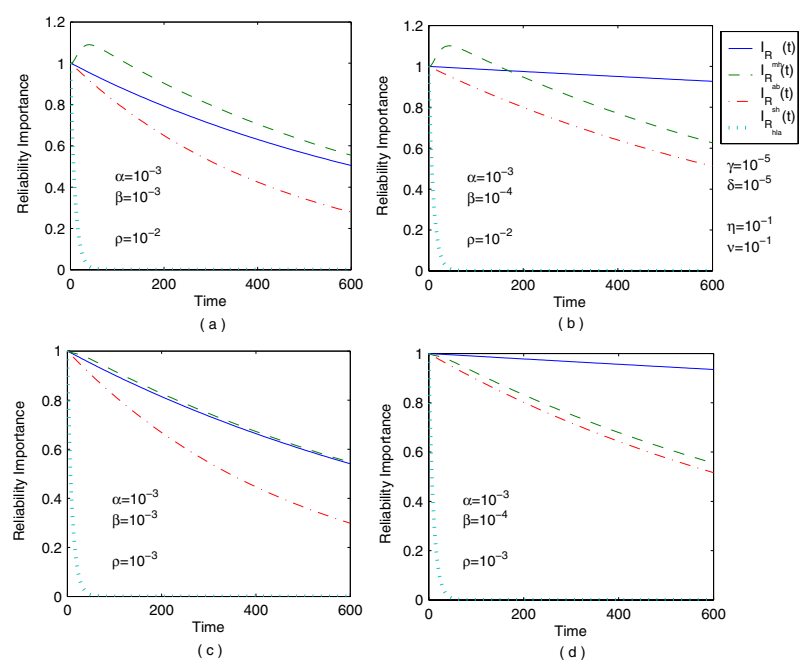

(d)
Figure 7. Two-terminal MTTF of SM scheme

reliability will start to decrease. We note that $\alpha, \beta$ and $\gamma$ express the similar effects on the MTTF of the MS scheme as that of the SM scheme. However, the MTTF varies little with $\delta$ as $\delta$ is only taken into consideration in state $c$ and state $c$ does not contribute much to the expected-reliability, which means that any gains by improving the reliability of the HLA will be small. This observation, however, is drawn with the caveat that we assume the HLA to only provide the location forward function. If it is required to provide other important functions, such as location update and discovery, then the analysis results would have been different. Finally, $\nu$ has the same behavior with $\eta$, as shown in Figure 7(d), which indicates that the location forward processing should also be done as quickly as possible when the handoff rate is high.

We have seen that the $\mathrm{MH}$ and the $\mathrm{AB}$ behave almost the same in the improvement gains in terms of the MTTF in the MS and the SM schemes. Now we observe them from another point of view to see whether this result will change or not. We define time-dependent reliability importance with respect to the expected-reliability to identify the relative importance of each component in a system. The time-dependent reliability importance (RI), $I_{R_{i}}$, of component $i, i=m h, a b, s h$, or $h l a$, is given by

$$
\begin{aligned}
I_{R_{i}}(t)=\frac{\partial E R(t)}{\partial R_{i}(t)}= & \sum_{s} Q_{s}(t) \cdot k_{i}(s)\left[R_{i}(t)\right]^{k_{i}(s)-1} \\
& \cdot \prod_{c}\left[R_{c}(t)\right]^{k_{c}(s)}, \quad c \neq i, \quad \text { (7) }
\end{aligned}
$$

as we substitute $E R(t)$ with Equation (1) and express $R_{s}(t)$ as $\prod_{c}\left[R_{c}(t)\right]^{k_{c}(s)}$. Applying Equation (7) in the SM

\section{Figure 8. Reliability importance of SM scheme}

scheme, we show the results in Figure 8. When the handoff rate is relatively high (Figure 8(a) and (b)), the RI of the $\mathrm{AB}$ increases first and then decreases, indicating the contributions of states $e$ and $f$ are high. If the $\mathrm{AB}$ and the $\mathrm{MH}$ experience the same failure rate (Figure 8(a)), the AB always gets the higher RI than the MH does. On the other hand, if the $\mathrm{AB}$ is more reliable than the $\mathrm{MH}$ (Figure 8(b)), the $\mathrm{AB}$ gets the higher RI initially and then the MH gets the higher RI; otherwise, the MH always gets the higher RI. This shows that the relative RIs of different components may vary with the intended working time of the system. We compare the difference between Figure 8(a) and (c), in which the $\mathrm{AB}$ and the $\mathrm{MH}$ inherit the same failure rate, so do the SH and the HLA. The difference between the RI of the $\mathrm{AB}$ and that of the $\mathrm{MH}$ is relatively large when the handoff rate is relatively high even they experience the same failure rate; however, they gets almost the same RI when the handoff rate is relatively low. The SH gets the higher RI than the HLA does despite of the handoff rate. All these are induced by the probabilities of different system states in which each component engages. When the handoff rate is high, the system gets greater probabilities in states $e$ and $f$, in which two ABs are employed. Therefore, the RI of the $\mathrm{AB}$ will be higher than that of the $\mathrm{MH}$. The $\mathrm{SH}$ is present in each system state, but the HLA only appears in state $c$. Obviously then, the RI of the SH should always be higher than that of the HLA.

\subsection{Scheme}

The system becomes more complicated in the MM scheme as both MHs may undergo handoffs, and the following invocation forwarding mechanisms also complicate 


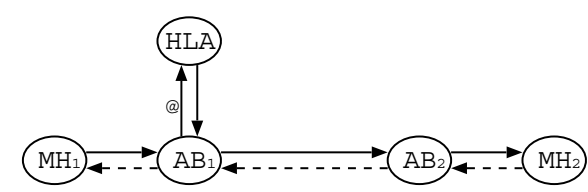

(h)

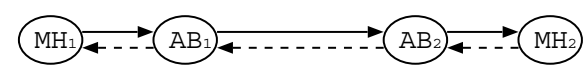

(i)

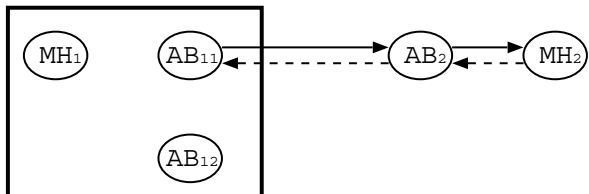

(j)

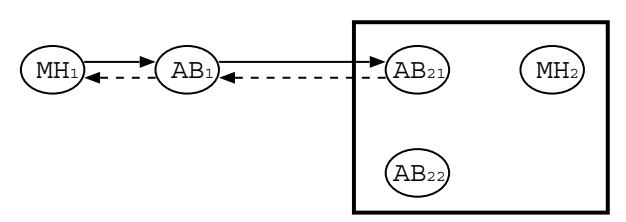

(k)

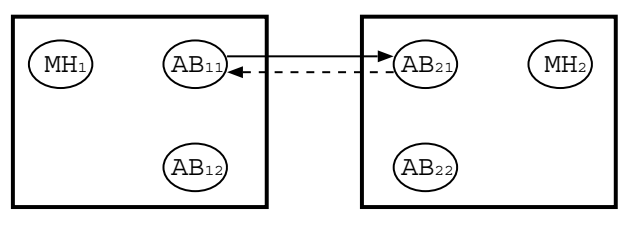

(1)

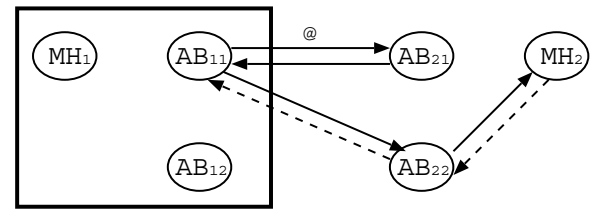

(m)

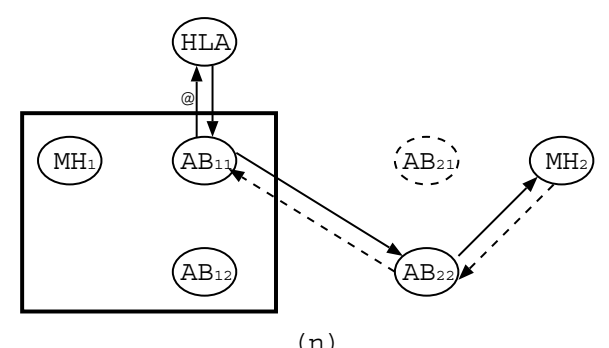

(n)

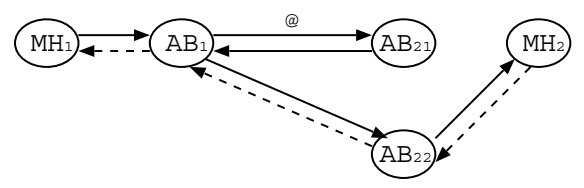

(o)

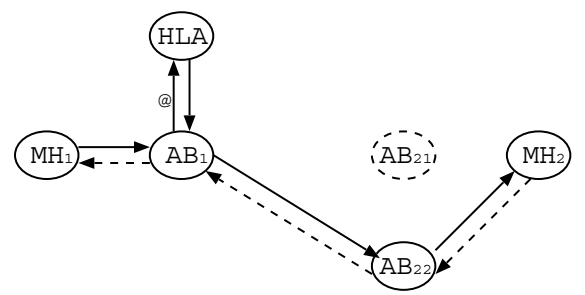

(p)

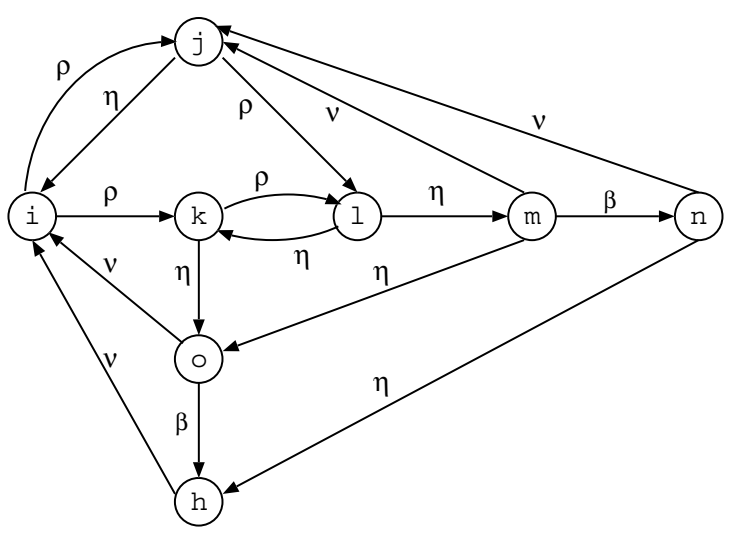

(III)

Figure 9. System states and Markov model in MM scheme 
the system states. The system states and the corresponding Markov model are shown in Figure 9. States $j$ and $k$ denote the system states in which only one MH is in handoff. There exists a probability that both MHs are in handoff, shown in state $l$. Here we assume that two MHs do not reside within the cell of the same $\mathrm{AB}$. This is reasonable because we could regard the derived results as the lower bounds and the difference is small. The variations of the probabilities of system states with time $t$ are shown in Figure 10 by employing that the system is initially in state $h$ with probability 1 . The probabilities of states $j, k$, and $o$ are on the same level of magnitude, and the probabilities of states $l, m$, and $n$ are on the other same level of magnitude. $Q_{l}(t) R_{l}(t), Q_{m}(t) R_{n}(t)$ and $Q_{n}(t) R_{n}(t)$ are omitted as they are too small to be depicted in the same figure. Actually, the system may enter other states not shown in Figure 9, such as that the $M H_{1}$ is in the location-forwarding period and the $\mathrm{MH}_{2}$ is in the handoff period at the same time. However, the probabilities of these states are as low as those of state $l, m$, and $n$, and their contributions to the expected-reliability are also negligible. The effects of different parameters on the MTTF and the RI in the MM scheme are very similar to those analyzed and presented in the SM scheme and thus omitted here.
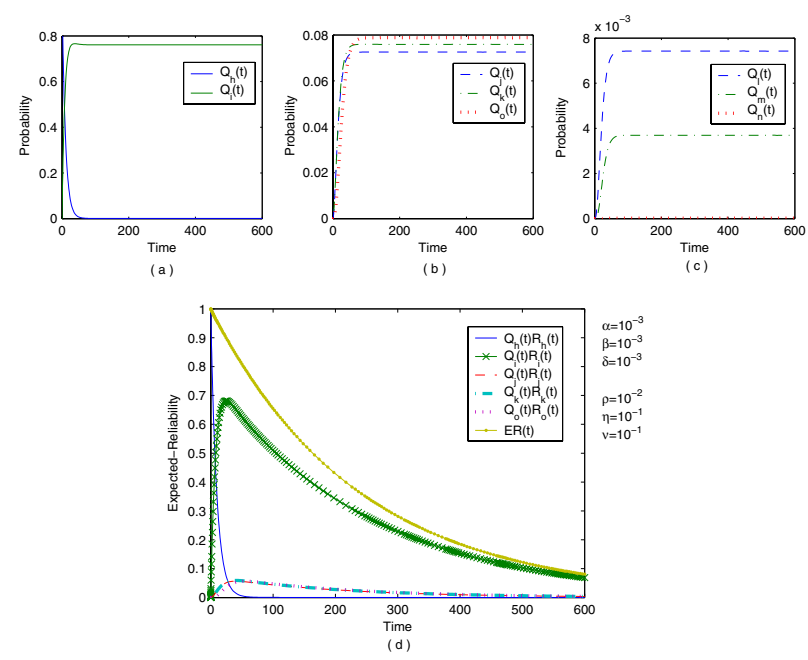

Figure 10. State probability and expectedreliability of MM scheme

\subsection{General Two-Terminal MTTF}

We have discussed the two-terminal MTTF with specific terminals in four communication schemes so far. Now we turn our attention to the general two-terminal MTTF of a wireless communication system which includes $n_{m}$ MHs and $n_{s}$ SHs. If each $\mathrm{MH}$ or $\mathrm{SH}$ has the same probability to initiate a communication, then the general two-terminal MTTF can be expressed by

$$
\begin{aligned}
M T T F & =\frac{1}{2 n_{m} n_{s}+\left(\begin{array}{c}
n_{m} \\
2
\end{array}\right)+\left(\begin{array}{c}
n_{s} \\
2
\end{array}\right)}\left[n_{m} n_{s} \cdot M T T F_{m s}\right. \\
& +n_{s} n_{m} \cdot M T T F_{s m}+\left(\begin{array}{c}
n_{m} \\
2
\end{array}\right) \cdot M T T F_{m m} \\
& \left.+\left(\begin{array}{c}
n_{s} \\
2
\end{array}\right) \cdot M T T F_{s s}\right]
\end{aligned}
$$

in which we assume that all the MHs share a common HLA.

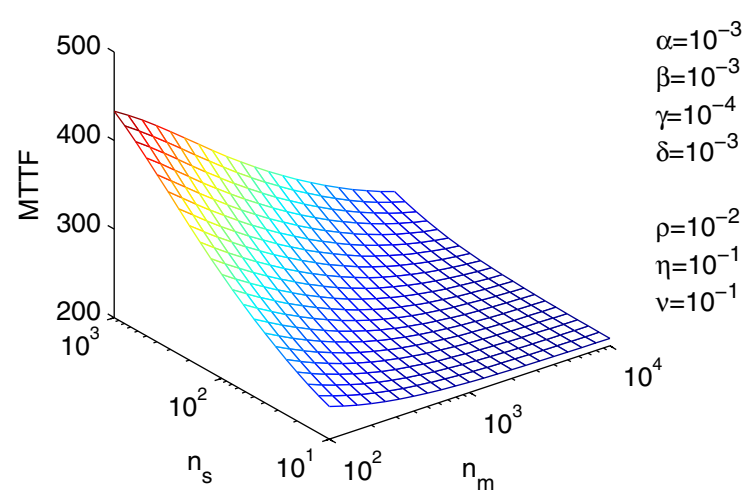

\section{Figure 11. General two-terminal MTTF vs. number of components}

Figure 11 shows how the general two-terminal MTTF varies with the number of nodes. As expected, the MTTF decreases with the number of MHs; however, it increases with the number of SHs. The MTTF $F_{s m}$ or $M T T F_{m s}$ is larger than the $M T T F_{m m}$ under the same parameter values as more components are engaged in the MM scheme. The more the number of SHs is, the more probably an $\mathrm{MH}$ communicates with an SH and the higher the MTTF is. The number of ABs may also affect the MTTF because the MH needs $\mathrm{ABs}$ to relay messages. According to our definition of the general two-terminal MTTF, however, the number of ABs has no effect on it.

\section{Conclusions}

Although a significant amount of research has been conducted in protocols, mobility models, and location management issues for wireless networks, little attention has been paid towards their reliability evaluation. In this paper, we extend traditional reliability analysis of wired networks to wireless networks with imperfect components. The unique characteristic of wireless networks is handoff, which leads to different communication structures in which 
various types and numbers of components are utilized. Traditional reliability definition is not suitable to be employed in this situation, so we propose a new term, two-terminal expected-reliability, to describe reliability scenarios in wireless networks. Based on this definition, we have made several interesting observations. First, the failure parameters of $\mathrm{MH}, \mathrm{AB}$, and SH behave similarly on the MTTF; however the failure parameter of HLA takes little effect on the MTTF. Second, if the handoff happens frequently, we should improve the performance of the handoff completion and the location forwarding mechanism; otherwise, the MTTF will decrease very quickly. Third, the general two-terminal MTTF increases with the number of SHs but decreases with the number of MHs. Finally, we have identified the reliability importance of each component with respect to the expected-reliability. Under different conditions different components in a wireless networks should be focused on to receive the highest reliability gain. Our investigation provides an initial yet overall approach to achieve this goal with several numerical examples.

\section{Acknowledgements}

The work described in this paper was fully supported by a grant from the Research Grants Council of the Hong Kong Special Administrative Region, China (Project No. CUHK4182/03E).

\section{References}

[1] K. K. Aggarwal, J. S. Gupta, and K. B. Misra. A simple method for reliabiilty evaluation of a communication system. IEEE Transactions on Communications, 23(5):563566, May 1975.

[2] S. Alagra, R. Rajagopalan, and S. Venkatesan. Tolerating mobile support station failures. In Proc. the 1st Conference on Fault Tolerant Systems, pages 225-231, Madras, India, Dec. 1995.

[3] S. Biaz and N. H. Vaidya. Tolerating visitor location register failures in mobile environments. In Proc. the 17th Symposium on Reliable Distributed Systems, pages 109-117, Oct. 1998.

[4] X. Chen and M. R. Lyu. Message logging and recovery in wireless CORBA using access bridge. In Proc. the 6th International Symposium on Autonomous Decentralized Systems, pages 107-114, Pisa, Italy, Apr. 2003.

[5] X. Chen and M. R. Lyu. Performance and effectiveness analysis of checkpointing in mobile environments. In Proc. the 22nd Symposium on Reliable Distributed Systems, pages 131-140, Florence, Italy, Oct. 2003.

[6] D. C. Cox. Wireless personal communications: What is it? IEEE Personal Communications Magazine, 2(2):20-35, Apr. 1995.

[7] W.-J. Ke and S.-D. Wang. Reliability evaluation for distributed computing networks with imperfect nodes. IEEE Transactions on Reliability, 46(3):342-349, Sept. 1997.
[8] P. Kubat. Estimation of reliability for communication/computer networks - simulation/analytic approach. IEEE Transactions on Communications, 37(9):927-933, Sept. 1989.

[9] W. Kuo and M. J. Zuo. Optimal Reliability Modeling: Principles and Applications. John Wiley \& Sons Inc., New Jersey, 2003.

[10] R. B. Marks, I. C. Gifford, and B. O'Hara. Standards in ieee 802 unleash the wireless internet. IEEE Microwave Magazine, 2(2):46-56, June 2001.

[11] V. A. Netes and B. P. Filin. Consideration of node failures in network-reliability calculation. IEEE Transactions on Reliability, 45(1):127-128, Mar. 1996.

[12] N. Neves and W. K. Fuchs. Adaptive recovery for mobile environments. Communications of the ACM, 40(1):68-74, Jan. 1997.

[13] Object Management Group. Wireless access and terminal mobility in CORBA, V1.0. OMG Document formal/03-0664, Mar. 2003.

[14] T. Park and H. Y. Yeom. An asynchronous recovery scheme based on optimistic message logging for the mobile computing systems. In Proc. the 20th International Conference on Distributed Computing Systems, pages 436-443, Taipei, Taiwan, Apr. 2000.

[15] D. K. Pradhan, P. Krishna, and N. H. Vaidya. Recoverable mobile environment: Design and trade-off analysis. In Proc. the 26th Annual International Symposium on Fault-Tolerant Computing, pages 16-25, Sendai, Japan, June 1996.

[16] G. Ray and J. Dunsmore. Reliability of network topologies. In Proc. the 7th IEEE Conference on Computer Communications, pages 842-850, New Orleans, Mar. 1988.

[17] J. Shaio. A family of algorithms for network reliability problems. In Proc. IEEE International Conference on Communications, volume 4, pages 2167-2173, New York City, May 2002.

[18] A. M. Shooman and A. Kershenbaum. Methods for communication-network reliability analysis: Probabilistic graph reduction. In Proc. Annual Reliability and Maintainability Symposium, pages 21-23, Las Vegas, Nevada, Jan. 1992.

[19] M. L. Shooman. Reliability of Computer Systems and Networks: Fault Tolerance, Analysis, and Design. John Wiley \& Sons Inc., New York, 2002.

[20] A. P. Snow, U. Varshney, and A. D. Malloy. Reliability and survivability of wireless and mobile networks. IEEE Computer, 33(7):49-55, July 2000.

[21] D. Torrieri. Calculation of node-pair reliability in large networks with unreliable nodes. IEEE Computer, 43(3):375377, Sept. 1994.

[22] U. Varshney, A. P. Snow, and A. D. Malloy. Measuring the reliability and survivability of infrastructure-oriented wireless networks. In Proc. the 26th Annual IEEE Conference on Local Computer Networks, pages 611-618, Tampa, Florida, Nov. 2001. 